\title{
On the Pricing of Storable Commodities
}

\author{
Dorje C. Brody ${ }^{1,2}$, Lane P. Hughston ${ }^{3}$, Xun YanG ${ }^{4}$ \\ ${ }^{1}$ Department of Mathematics, University of Surrey, \\ Guildford GU2 7XH, United Kingdom \\ ${ }^{2}$ St Petersburg National Research University of Information Technologies, \\ Mechanics and Optics, 49 Kronverksky Avenue, St Petersburg 197101, Russia \\ ${ }^{3}$ Department of Computing, Goldsmiths University of London, \\ New Cross, London SE14 6NW, United Kingdom \\ ${ }^{4}$ Shell International Limited, Shell Centre, London SE1 7NA, United Kingdom
}

(Dated: December 1, 2021)

\begin{abstract}
This paper introduces an information-based model for the pricing of storable commodities such as crude oil and natural gas. The model uses the concept of market information about future supply and demand as a basis for valuation. Physical ownership of a commodity is taken to provide a stream of convenience dividends equivalent to a continuous cash flow. The market filtration is assumed to be generated jointly by (i) current and past levels of the dividend rate, and (ii) partial information concerning the future of the dividend flow. The price of a commodity is the expectation under a suitable pricing measure of the totality of the discounted risk-adjusted future convenience dividend, conditional on the information provided by the market filtration. In the situation where the dividend rate is modelled by an Ornstein-Uhlenbeck process, the prices of options on commodities can be derived in closed form. The approach that we present can be applied to other assets that yield potentially negative effective cash flows, such as real estate, factories, refineries, mines, and power generating plants.
\end{abstract}

Keywords: Commodity markets; commodity derivatives; crude oil; natural gas; convenience yield; information-based asset pricing; market microstructure.

To appear as Chapter 17 in Financial Informatics: An Information-Based Approach to Asset Pricing. D. C. Brody, L. P. Hughston \& A. Macrina (editors). Singapore: World Scientific Publishing Company (2022).

\section{INTRODUCTION}

In the extensive literature devoted to the pricing and risk management of commodity derivatives, most investigations take as a starting point the specification of a dynamical model for the price process for the commodity. The outcome of chance in the market in which the commodity is traded is usually represented in such studies with the specification of a fixed probability space equipped with the filtration generated by a Brownian motion of one or more dimensions, and it is typically assumed that the commodity price can be modelled as an Ito process adapted to this filtration. Such an approach to the pricing of commodities and related derivatives is in line with the "standard" modelling framework for asset pricing within which much or event most of modern finance theory has been pursued [1, 2].

Nevertheless, there is a fundamental methodological issue in the standard framework: namely, that the market filtration is fixed in an essentially ad hoc way, and that no indi- 
cation is provided concerning the nature of the information it purports to convey, or why it is relevant to the price. The information is in practice normally no more than that of the price movements themselves, so it can hardly be claimed in any useful way that the price movements are taking place "in response" to "shocks" associated with the arrival of information, for the shocks, as we have said, are no more than the asset price movements themselves.

One knows, however, that in real markets, information concerning the possible future cash flows and other benefits or obligations linked to the physical possession of an asset can be crucial in the determination of trading decisions, even in situations where such information is imperfect. The movement of the price of an asset should thus be regarded as a derived concept, induced by the flow of information to market participants. This is the point of view put forward in the information-based asset pricing theory of Brody, Hughston \& Macrina [3 5] that forms the analytical basis of the present investigation; see also [6, 7].

The goal of this paper is to incorporate the role played by forward-looking information in commodity markets in such a context, and to derive a model for the prices used as underlyings in the valuation of commodity derivatives. Specifically, we make use of the concept of market information about future supply and demand as a basis for the valuation of storable commodities. The theory of commodity pricing from a modern perspective has a long history, starting from the work of Black [8] and Brennan \& Schwartz [9]. For a detailed treatment of theory of storage, convenience yield, and related concepts, the reader can be referred to Geman [10] and references cited therein. In our approach, we shall assume that the possession of one standard unit of a commodity provides a net "convenience dividend" equivalent to a cash flow $\left\{X_{t}\right\}_{t \geq 0}$. We thus work directly with the actual flow of benefit arising from the possession of the commodity, rather than the percentage convenience yield. The point is that the percentage convenience yield so often used in commodity modelling is a secondary notion, since it depends on the price, which is what we are trying to determine. In what follows, we present a simple model for the convenience dividend process $\left\{X_{t}\right\}$. Additionally, we introduce a market information process $\left\{\xi_{t}\right\}_{t \geq 0}$ that provides partial or speculative information about the future dividend flow. The market filtration is then assumed to be generated jointly by these two processes. In that sense, we are explicitly constructing the market filtration in such a way that it contains information relevant to the commodity price. Given the market filtration, the price of the commodity is taken to be the risk-adjusted discounted expected value of the totality of the future convenience dividends. We model $\left\{X_{t}\right\}$ by an Ornstein-Uhlenbeck process. We model $\left\{\xi_{t}\right\}$ by a process that consists of two terms: a "signal" term containing information about the future convenience dividend flow, and a "noise" term given by an independent Brownian motion. By use of this information-based model we are able to derive closed-form expressions for both the price of the commodity and for the prices of associated derivatives.

The remainder of the paper is organized as follows. In $\llbracket$ we introduce our model for the net convenience dividend and for the market filtration. In $\$$ III various useful facts relating to the Ornstein-Uhlenbeck (OU) process are recalled - in particular, certain features of the OU bridge. These are used in $\$ \mathrm{IV}$ to show in Proposition 1 that the information process and the convenience dividend rate are jointly Markovian, and in $8 \mathrm{~V}$ to derive an expression for the commodity price. Finally, in $\$ \mathrm{VI}$ we present pricing formulae for call options on the underlying spot price. 


\section{INFORMATION-BASED COMMODITY PRICING}

In the information-based approach of Brody, Hughston \& Macrina [3 -5], the starting point is the specification of (i) a set of one or more random variables (called "market factors") determining the cash flows associated with a given asset, and (ii) a set of one or more random processes (called "information processes") determining the flow of information to market participants concerning these market factors. The setup, more specifically, is as follows. We model the outcome of chance in a commodity market with the specification of a probability space $(\Omega, \mathcal{F}, \mathbb{Q})$. The market is not assumed to be complete, but we do assume the existence of a preferred pricing measure (or "risk neutral" measure) $\mathbb{Q}$. In more detail, we assume the existence of a physical measure and a pricing kernel that with respect to the physical measure takes the form of a discount factor times a strictly positive martingale. The martingale is used to change the measure to $\mathbb{Q}$, and from that point onward we formulate the details of the theory with reference to that measure. Then if $\left\{X_{t}\right\}$ represents the net convenience dividend, which is given by the benefits associated with possession of the commodity less storage costs and any other direct costs associated with the said possession, the price of the commodity at time $t$ is given by

$$
S_{t}=\frac{1}{\pi_{t}} \mathbb{E}\left[\int_{t}^{\infty} \pi_{u} X_{u} \mathrm{~d} u \mid \mathcal{F}_{t}\right]
$$

where the expectation is taken under $\mathbb{Q}$. Here the discount factor $\left\{\pi_{t}\right\}_{t \geq 0}$ is given in terms of the short rate $\left\{r_{t}\right\}_{t \geq 0}$ by

$$
\pi_{t}=\exp \left(-\int_{0}^{t} r_{s} \mathrm{~d} s\right)
$$

The associated money-market account process is then given by $\left\{1 / \pi_{t}\right\}_{t \geq 0}$. For simplicity, we assume that the default-free interest rate system is deterministic. The market filtration $\left\{\mathcal{F}_{t}\right\}$, with respect to which the conditioning is taken in (1), is taken to be generated jointly by (a) the convenience dividend process $\left\{X_{t}\right\}_{t \geq 0}$ and (b) a market information process $\left\{\xi_{t}\right\}_{t \geq 0}$ of the form

$$
\xi_{t}=\sigma t \int_{t}^{\infty} \pi_{u} X_{u} \mathrm{~d} u+B_{t}
$$

representing partial or noisy information about the future dividend flow. The parameter $\sigma$ determines the rate at which information about the future dividend stream is revealed to the market. The $\mathbb{Q}$-Brownian motion $\left\{B_{t}\right\}$ represents noise arising from rumour, baseless speculation, uninformed trading, fake news, and the like, and is assumed to be independent of the dividend process $\left\{X_{t}\right\}$. Therefore for each $t \geq 0$ we have

$$
\mathcal{F}_{t}=\sigma\left[\left\{\xi_{s}\right\}_{0 \leq s \leq t},\left\{X_{s}\right\}_{0 \leq s \leq t}\right] .
$$

The next step is to specify the form of the dividend process. We consider in this paper the case in which $\left\{X_{t}\right\}$ is an Ornstein-Uhlenbeck (OU) process. It is worth recalling, by way of contrast, that Gibson \& Schwartz [11, 12] assume that the percentage convenience yield should follow a mean-reverting process, an approach that has been followed in many subsequent works [13 20]. The idea of the present work, however, is that a mean-reverting 
absolute convenience dividend reflects the notion that in the long term there is an equilibrium rate of benefit obtained by storing or holding the commodity. We thus assume that the dividend process satisfies a stochastic equation of the form

$$
\mathrm{d} X_{t}=\kappa\left(\theta-X_{t}\right) \mathrm{d} t+\psi \mathrm{d} \beta_{t},
$$

where $\left\{\beta_{t}\right\}$ is a $\mathbb{Q}$-Brownian motion that is independent of $\left\{B_{t}\right\}$. We allow for the possibility that the dividend rate may occasionally be negative. The mean reversion level $\theta$, the reversion rate $\kappa$, and the dividend volatility $\psi$ are assumed to be constant in the present discussion, although the results can be readily generalized to the time-dependent case.

\section{PROPERTIES OF THE ORNSTEIN-UHLENBACK PROCESS}

Before we proceed to work out the conditional expectation (11), it will be useful to comment on various properties of the Ornstein-Uhlenbeck process. These properties, some of which will be well known, but others perhaps less so, will help us simplify the calculations. It is an elementary exercise to check that the solution to (5) takes the form

$$
X_{t}=\mathrm{e}^{-\kappa t} X_{0}+\theta\left(1-\mathrm{e}^{-\kappa t}\right)+\psi \mathrm{e}^{-\kappa t} \int_{0}^{t} \mathrm{e}^{\kappa s} \mathrm{~d} \beta_{s} .
$$

The Ornstein-Uhlenbeck process has the property that if we "reinitialize" the process at time $t$ then its value at some later time $T>t$ can be expressed as

$$
X_{T}=\mathrm{e}^{-\kappa(T-t)} X_{t}+\theta\left(1-\mathrm{e}^{-\kappa(T-t)}\right)+\psi \mathrm{e}^{-\kappa T} \int_{t}^{T} \mathrm{e}^{\kappa u} \mathrm{~d} \beta_{u} .
$$

Since $\left\{X_{t}\right\}$ is a Gaussian process, one can verify the following by use of covariance relations:

Lemma 1 The random variables $X_{t}$ and $X_{T}-\mathrm{e}^{-\kappa(T-t)} X_{t}$ are independent.

Proof. The mean of $X_{t}$ is given by

$$
\mathbb{E}\left[X_{t}\right]=\mathrm{e}^{-\kappa t} X_{0}+\theta\left(1-\mathrm{e}^{-\kappa t}\right) .
$$

A straightforward calculation using the Ito isometry then shows that

$$
\operatorname{Var}\left[X_{t}\right]=\frac{\psi^{2}}{2 \kappa}\left(1-\mathrm{e}^{-2 \kappa t}\right)
$$

and that for $0 \leq t \leq T$ we have

$$
\operatorname{Cov}\left[X_{t}, X_{T}\right]=\frac{\psi^{2}}{\kappa} \mathrm{e}^{-\kappa T} \sinh \kappa t,
$$

from which it follows that

$$
\operatorname{Cov}\left[X_{t}, X_{T}-\mathrm{e}^{-\kappa(T-t)} X_{t}\right]=0,
$$

and hence the claimed independence. 
This property of the OU process corresponds to an orthogonal decomposition of the form

$$
X_{T}=\left(X_{T}-\mathrm{e}^{-\kappa(T-t)} X_{t}\right)+\mathrm{e}^{-\kappa(T-t)} X_{t}
$$

for $T>t$. The validity of Lemma 1 can also be checked by direct inspection of (6) and (7). It should be evident that if the reversion rate is set to zero, then (12) reduces to the independent-increments decomposition of a Brownian motion.

Interestingly, there is another orthogonal decomposition of the OU process that is somewhat less obvious than (12). This is given by the identity

$$
X_{t}=\left(X_{t}-\frac{\sinh \kappa t}{\sinh \kappa T} X_{T}\right)+\frac{\sinh \kappa t}{\sinh \kappa T} X_{T} .
$$

The process $\left\{b_{t T}\right\}_{0 \leq t \leq T}$, defined for fixed $T$ by

$$
b_{t T}=X_{t}-\frac{\sinh \kappa t}{\sinh \kappa T} X_{T}
$$

appearing in (13), is an Ornstein-Uhlenbeck (OU) bridge. The OU bridge interpolates between the fixed values $b_{0 T}=X_{0}$ and $b_{T T}=0$, and we are led to another useful result.

In particular, a calculation shows the following:

Lemma 2 The Ornstein-Uhlenbeck bridge $\left\{b_{t T}\right\}_{0 \leq t \leq T}$ and the random variable $X_{U}$ are independent for all $T$ and $U$ such that $0 \leq T \leq U$.

Proof. Since $\left\{b_{t T}\right\}_{0 \leq t \leq T}$ and $X_{U}$ are jointly Gaussian, it suffices to show that for any choice of $t, T, U$ such that $t \leq T \leq U$ the random variables $b_{t T}$ and $X_{U}$ are independent. We have

$$
\operatorname{Cov}\left[X_{t}, X_{U}\right]=\frac{\psi^{2}}{\kappa} \mathrm{e}^{-\kappa U} \sinh \kappa t
$$

and

$$
\operatorname{Cov}\left[X_{T}, X_{U}\right]=\frac{\psi^{2}}{\kappa} \mathrm{e}^{-\kappa U} \sinh \kappa T,
$$

from which it follows that

$$
\operatorname{Cov}\left[X_{t}-\frac{\sinh \kappa t}{\sinh \kappa T} X_{T}, X_{U}\right]=0,
$$

and hence the claimed independence.

We note that the mean and variance of the OU bridge are given, respectively, by

$$
\mathbb{E}\left[b_{t T}\right]=\frac{\sinh \kappa(T-t)}{\sinh \kappa T} X_{0}+\left[1-\frac{\sinh \kappa t+\sinh \kappa(T-t)}{\sinh \kappa T}\right] \theta
$$

and

$$
\operatorname{Var}\left[b_{t T}\right]=\frac{\psi^{2}}{\kappa} \sinh \kappa t\left[\cosh \kappa t-\frac{\sinh \kappa t}{\sinh \kappa T} \cosh \kappa T\right] .
$$




\section{MARKOV PROPERTY OF MARKET INFORMATION}

When working with conditional expectations, we often use the shorthand $\mathbb{E}[Y \mid Z]$ in place of $\mathbb{E}[Y \mid \sigma\{Z\}]$, where $\sigma\{Z\}$ denotes the $\sigma$-algebra generated by $Z$. Keeping in mind this notation, we frequently make use of the following. Let $X, Y$, and $Z$ be random variables, and assume that $X$ is integrable. Then if $\sigma\{X, Y\}$ and $\sigma\{Z\}$ are independent it holds that

$$
\mathbb{E}[X \mid Y, Z]=\mathbb{E}[X \mid Y]
$$

See, for example, Williams [21], section 9.7. We proceed to work out the conditional expectation in (II) to determine the commodity price. The following result will facilitate the calculations.

Proposition 3 The information process $\left\{\xi_{t}\right\}$ and the dividend rate $\left\{X_{t}\right\}$ are jointly Markov.

Proof. We need to show that for $0 \leq t \leq u$ it holds that

$$
\mathbb{Q}\left[\xi_{u}<a \cap X_{u}<b \mid\left\{\xi_{s}\right\}_{0 \leq s \leq t},\left\{X_{s}\right\}_{0 \leq s \leq t}\right]=\mathbb{Q}\left[\xi_{u}<a \cap X_{u}<b \mid \xi_{t}, X_{t}\right] .
$$

Let us define a process $\left\{\eta_{t}\right\}$ by setting

$$
\eta_{t}=\sigma t \int_{0}^{\infty} \pi_{u} X_{u} \mathrm{~d} u+B_{t}
$$

It should be evident that

$$
\sigma\left[\left\{\xi_{s}\right\}_{0 \leq s \leq t},\left\{X_{s}\right\}_{0 \leq s \leq t}\right]=\sigma\left[\left\{\eta_{s}\right\}_{0 \leq s \leq t},\left\{X_{s}\right\}_{0 \leq s \leq t}\right] .
$$

This follows from the fact that

$$
\eta_{t}=\xi_{t}+\sigma t \int_{0}^{t} \pi_{u} X_{u} \mathrm{~d} u
$$

We observe that $\left\{\eta_{t}\right\}$ is Markov in its own filtration. To see this, it suffices to verify that

$$
\mathbb{Q}\left(\eta_{t} \leq x \mid \eta_{s}, \eta_{s_{1}}, \eta_{s_{2}}, \ldots, \eta_{s_{k}}\right)=\mathbb{Q}\left(\eta_{t} \leq x \mid \eta_{s}\right)
$$

for any collection of times $t, s, s_{1}, s_{2}, \ldots, s_{k}$ such that $t \geq s \geq s_{1} \geq s_{2} \geq \cdots \geq s_{k}>0$. Now, it is an elementary property of Brownian motion that for any times $t, s, s_{1}$ satisfying $t>s>s_{1}>0$ the random variables $B_{t}$ and $B_{s} / s-B_{s_{1}} / s_{1}$ are independent. More generally, for $s>s_{1}>s_{2}>s_{3}>0$, we find that $B_{s} / s-B_{s_{1}} / s_{1}$ and $B_{s_{2}} / s_{2}-B_{s_{3}} / s_{3}$ are independent. Observing that $\eta_{s} / s-\eta_{s_{1}} / s_{1}=B_{s} / s-B_{s_{1}} / s_{1}$, we find that

$$
\begin{aligned}
\mathbb{Q}\left(\eta_{t} \leq x \mid \eta_{s}, \eta_{s_{1}}, \ldots, \eta_{s_{k}}\right) & =\mathbb{Q}\left(\eta_{t} \leq x \mid \eta_{s}, \frac{\eta_{s}}{s}-\frac{\eta_{s_{1}}}{s_{1}}, \cdots, \frac{\eta_{s_{k-1}}}{s_{k-1}}-\frac{\eta_{s_{k}}}{s_{k}}\right) \\
& =\mathbb{Q}\left(\eta_{t} \leq x \mid \eta_{s}, \frac{B_{s}}{s}-\frac{B_{s_{1}}}{s_{1}}, \cdots, \frac{B_{s_{k-1}}}{s_{k-1}}-\frac{B_{s_{k}}}{s_{k}}\right) .
\end{aligned}
$$


But since $\eta_{t}$ and $\eta_{s}$ are jointly independent of $B_{s} / s-B_{s_{1}} / s_{1}, \cdots$, the Markov property (25) follows for $\left\{\eta_{t}\right\}$. Let us now define

$$
\mathcal{G}_{t}=\sigma\left(\left\{\frac{\eta_{t}}{t}-\frac{\eta_{s}}{s}\right\}_{0<s \leq t}\right) .
$$

Then clearly we have

$$
\mathcal{G}_{t}=\sigma\left(\left\{\frac{B_{t}}{t}-\frac{B_{s}}{s}\right\}_{0<s \leq t}\right),
$$

that is to say, $\mathcal{G}_{t}$ is generated by the Brownian bridge underlying the noise component of the information process. Note that the sigma algebras $\sigma\left[\eta_{t},\left\{X_{s}\right\}\right]$ and $\mathcal{G}_{t}$ are independent. As a consequence, writing

$$
F\left[a, b \mid \mathcal{F}_{t}\right]=\mathbb{P}\left[\xi_{u}<a \cap X_{u}<b \mid \mathcal{F}_{t}\right]
$$

for the conditional bivariate distribution function, we have

$$
\begin{aligned}
F\left[a, b \mid \mathcal{F}_{t}\right] & =\mathbb{E}\left[\mathbb{1}\left(\xi_{u}<a\right) \mathbb{1}\left(X_{u}<b\right) \mid \mathcal{F}_{t}\right] \\
& =\mathbb{E}\left[\mathbb{1}\left(\xi_{u}<a\right) \mathbb{1}\left(X_{u}<b\right) \mid\left\{\xi_{s}\right\}_{0 \leq s \leq t},\left\{X_{s}\right\}_{0 \leq s \leq t}\right] \\
& =\mathbb{E}\left[\mathbb{1}\left(\xi_{u}<a\right) \mathbb{1}\left(X_{u}<b\right) \mid\left\{\eta_{s}\right\}_{0 \leq s \leq t},\left\{X_{s}\right\}_{0 \leq s \leq t}\right] \\
& =\mathbb{E}\left[\mathbb{1}\left(\xi_{u}<a\right) \mathbb{1}\left(X_{u}<b\right) \mid \eta_{t}, \mathcal{G}_{t},\left\{X_{s}\right\}_{0 \leq s \leq t}\right] \\
& =\mathbb{E}\left[\mathbb{1}\left(\xi_{u}<a\right) \mathbb{1}\left(X_{u}<b\right) \mid \eta_{t},\left\{X_{s}\right\}_{0 \leq s \leq t}\right] \\
& =\mathbb{E}\left[\mathbb{1}\left(\xi_{u}<a\right) \mathbb{1}\left(X_{u}<b\right) \mid \xi_{t},\left\{X_{s}\right\}_{0 \leq s \leq t}\right] .
\end{aligned}
$$

On the other hand, recalling the definition of the OU bridge given by (14), we have

$$
\sigma\left[\xi_{t},\left\{X_{s}\right\}_{0 \leq s \leq t}\right]=\sigma\left[\xi_{t}, X_{t},\left\{b_{s t}\right\}_{0 \leq s \leq t}\right] .
$$

Now, it is easy to see that $\left\{b_{s t}\right\}_{0 \leq s \leq t}$ and $\left\{X_{u}\right\}_{u \geq t}$ are independent. It follows that $\sigma\left[\left\{b_{s t}\right\}_{0 \leq s \leq t}\right]$ and $\sigma\left[\xi_{t}, X_{t}, \xi_{u}, X_{u}\right]$ are independent, from which we get (21).

\section{COMMODITY PRICING FORMULA}

The joint Markov property (21) implies that

$$
\mathbb{E}\left[\int_{t}^{\infty} \pi_{u} X_{u} \mathrm{~d} u \mid\left\{\xi_{s}\right\}_{0 \leq s \leq t},\left\{X_{s}\right\}_{0 \leq s \leq t}\right]=\mathbb{E}\left[\int_{t}^{\infty} \pi_{u} X_{u} \mathrm{~d} u \mid \xi_{t}, X_{t}\right],
$$

which allows one to reduce the problem of working out the commodity price (1) to that of calculating

$$
S_{t}=\frac{1}{\pi_{t}} \mathbb{E}\left[\int_{t}^{\infty} \pi_{u} X_{u} \mathrm{~d} u \mid \xi_{t}, X_{t}\right] .
$$

One observes that from the orthogonal decomposition (12) we can isolate the dependence of the commodity price on the current level of the convenience dividend rate $X_{t}$. Remarkably, the dependence turns out to be linear. That is, we have

$$
\int_{t}^{\infty} \pi_{u} X_{u} \mathrm{~d} u=\int_{t}^{\infty} \pi_{u}\left(X_{u}-\mathrm{e}^{-\kappa(u-t)} X_{t}\right) \mathrm{d} u+\left(\int_{t}^{\infty} \pi_{u} \mathrm{e}^{-\kappa(u-t)} \mathrm{d} u\right) X_{t} .
$$


Substituting this formula in equation (33), we deduce that

$$
\begin{array}{r}
\pi_{t} S_{t}=\mathbb{E}\left[\int_{t}^{\infty} \pi_{u}\left(X_{u}-\mathrm{e}^{-\kappa(u-t)} X_{t}\right) \mathrm{d} u \mid \xi_{t}, X_{t}\right] \\
+\mathbb{E}\left[\left(\int_{t}^{\infty} \pi_{u} \mathrm{e}^{-\kappa(u-t)} \mathrm{d} u\right) X_{t} \mid \xi_{t}, X_{t}\right] \\
=\mathbb{E}\left[A_{t} \mid \xi_{t}, X_{t}\right]+\left(\int_{t}^{\infty} \pi_{u} \mathrm{e}^{-\kappa(u-t)} \mathrm{d} u\right) X_{t},
\end{array}
$$

where

$$
A_{t}=\int_{t}^{\infty} \pi_{u}\left(X_{u}-\mathrm{e}^{-\kappa(u-t)} X_{t}\right) \mathrm{d} u .
$$

Next we observe that as a consequence of (3) and (36) we have

$$
\xi_{t}=\sigma t\left[A_{t}+\left(\int_{t}^{\infty} \pi_{u} \mathrm{e}^{-\kappa(u-t)} \mathrm{d} u\right) X_{t}\right]+B_{t} .
$$

It follows that

$$
\pi_{t} S_{t}=\mathbb{E}\left[A_{t} \mid \sigma t A_{t}+B_{t}, X_{t}\right]+\left(\int_{t}^{\infty} \pi_{u} \mathrm{e}^{-\kappa(u-t)} \mathrm{d} u\right) X_{t} .
$$

Note that the conditioning with respect to $X_{t}$ in the first term above drops out since by Lemma (11) the random variables $\left\{X_{u}-\mathrm{e}^{-\kappa(u-t)} X_{t}\right\}_{u>t}$ and $X_{t}$ are independent, which allows one to deduce that the sigma-algebras $\sigma\left\{X_{t}\right\}$ and $\sigma\left\{A_{t}, B_{t}\right\}$ are independent. Therefore,

$$
\pi_{t} S_{t}=\mathbb{E}\left[A_{t} \mid \sigma t A_{t}+B_{t}\right]+\left(\int_{t}^{\infty} \pi_{u} \mathrm{e}^{-\kappa(u-t)} \mathrm{d} u\right) X_{t} .
$$

The problem of determining the commodity price is thus reduced to that of calculating a conditional expectation of the form $\mathbb{E}\left[A_{t} \mid A_{t}+C_{t}\right]$ for $t>0$, where $A_{t}$ is given by (36) and $C_{t}=B_{t} / \sigma t$. We observe that $A_{t}$ and $C_{t}$ are independent Gaussian random variables. To compute the conditional expectation above, we recall another result concerning orthogonal decompositions of Gaussian random variables:

Lemma 4 If $A$ and $C$ are independent Gaussian random variables, then $A+C$ and $(1-$ $z) A-z C$ are independent if $z=\operatorname{Var}[A] /(\operatorname{Var}[A]+\operatorname{Var}[C])$.

In view of this observation, let us express $A_{t}$ in the form

$$
A_{t}=z_{t}\left(A_{t}+C_{t}\right)+\left(1-z_{t}\right) A_{t}-z_{t} C_{t},
$$

where

$$
z_{t}=\frac{\operatorname{Var}\left[A_{t}\right]}{\operatorname{Var}\left[A_{t}\right]+\operatorname{Var}\left[C_{t}\right]}
$$

Then we find that

$$
\mathbb{E}\left[A_{t} \mid A_{t}+C_{t}\right]=z_{t}\left(A_{t}+C_{t}\right)+\left(1-z_{t}\right) \mathbb{E}\left[A_{t}\right]-z_{t} \mathbb{E}\left[C_{t}\right] .
$$


Clearly, we have $\mathbb{E}\left[C_{t}\right]=0$. Furthermore, if we set $T=u$ in equation (17) we deduce that

$$
\begin{aligned}
\mathbb{E}\left[A_{t}\right] & =\mathbb{E}\left[\theta \int_{t}^{\infty} \pi_{u}\left(1-\mathrm{e}^{-\kappa(u-t)}\right) \mathrm{d} u+\psi \int_{t}^{\infty} \mathrm{e}^{-\kappa u} \pi_{u} \int_{t}^{u} \mathrm{e}^{\kappa s} \mathrm{~d} \beta_{s} \mathrm{~d} u\right] \\
& =\theta \int_{t}^{\infty} \pi_{u} \mathrm{~d} u-\theta \int_{t}^{\infty} \pi_{u} \mathrm{e}^{-\kappa(u-t)} \mathrm{d} u .
\end{aligned}
$$

The final step in deriving the commodity price is to determine the variances of $A_{t}$ and $C_{t}$. To simplify the notation let us write

$$
p_{t}=\int_{t}^{\infty} \pi_{u} \mathrm{~d} u, \quad q_{t}=\int_{t}^{\infty} \pi_{u} \mathrm{e}^{-\kappa(u-t)} \mathrm{d} u .
$$

Then a short calculation shows that

$$
\operatorname{Var}\left[A_{t}\right]=\psi^{2} \int_{t}^{\infty} q_{s}^{2} \mathrm{~d} s, \quad \operatorname{Var}\left[C_{t}\right]=\frac{1}{\sigma^{2} t}
$$

and hence that

$$
z_{t}=\frac{\sigma^{2} \psi^{2} t \int_{t}^{\infty} q_{s}^{2} \mathrm{~d} s}{1+\sigma^{2} \psi^{2} t \int_{t}^{\infty} q_{s}^{2} \mathrm{~d} s}
$$

Putting these results together, we deduce that the price of the commodity at $t$ is given by

$$
\pi_{t} S_{t}=\left(1-z_{t}\right)\left[\theta p_{t}+q_{t}\left(X_{t}-\theta\right)\right]+\frac{1}{\sigma t} z_{t} \xi_{t} .
$$

Observe that the first term in (47) is essentially the annuity valuation of a constant dividend rate set at the reversion level $\theta$, together with a correction term to adjust for the present level of the dividend rate. The second term, on the other hand, represents the contribution from the noisy observation of the future dividend flow.

Several interesting observations can be made regarding the weight factor (46), which lies between zero and one for all $t$. For large $\psi$ and/or large $\sigma$, the value of $z_{t}$ tends to unity; for small $\psi$ and/or small $\sigma$, the value of $z$ tends to 0 . Hence, if the market information has a low noise content, or if the volatility of the convenience dividend is high, then market participants also rely heavily on the information available about the future in their determination of the price, rather than assuming that the current value of the dividend is a good indicator for the future.

Conversely, in the absence of a strong signal concerning the future dividend flow, an annuity valuation based on the current dividend level will dominate the price. We see therefore that important intuitive characteristics are encoded explicitly in the pricing formula (47). Indeed, (477) captures rather well the idea of information-based asset pricing, showing how varying amounts of information about the future can affect the development of prices, and that prices typically represent a kind of compromise between what we know for sure at some given time, and the less trustworthy but nevertheless significant intelligence that we may possess regarding events that lay ahead.

In the special case for which the interest rate is constant, the valuation formula (47) simplifies somewhat to give the following:

$$
S_{t}=\left(1-z_{t}\right) \frac{1}{r}\left[\frac{\kappa}{r+\kappa} \theta+\frac{r}{r+\kappa} X_{t}\right]+\frac{\mathrm{e}^{r t}}{\sigma t} z_{t} \xi_{t}
$$


where the weight factors are

$$
z_{t}=\frac{\sigma^{2} \psi^{2} t}{2 r(r+\kappa)^{2} \mathrm{e}^{2 r t}+\sigma^{2} \psi^{2} t}, \quad 1-z_{t}=\frac{2 r(r+\kappa)^{2} \mathrm{e}^{2 r t}}{2 r(r+\kappa)^{2} \mathrm{e}^{2 r t}+\sigma^{2} \psi^{2} t} .
$$

We have performed Monte Carlo simulation studies to gain further intuition concerning the dynamical behaviour of the commodity price. Furthermore, we have calibrated the model parameters to the prices of crude oil, and we have compared the resulting simulated sample paths to market data. In the case of the crude oil markets we are able to estimate the expected long-term future spot price from the historical average of spot prices, since there exists a supply-demand equilibrium price level to which the long-run price tends to converge. The results indicate that even in the constant-parameter model considered above, the model is sufficiently rich to capture elements of the behaviour of market data

\section{PRICING COMMODITY DERIVATIVES}

We now return to the price process (48) in the case of a constant interest rate and work out the value of a European-style call option with strike $K$ and maturity $T$. Since $\mathbb{Q}$ is the pricing measure, we have

$$
C_{0}=\mathrm{e}^{-r T} \mathbb{E}\left[\left(S_{T}-K\right)^{+}\right] .
$$

We observe that $S_{T}$ consists of a linear combination of three random components, namely $X_{T}, \int_{T}^{\infty} \mathrm{e}^{-r u} X_{u} \mathrm{~d} u$, and $B_{T}$, and that all three components are Gaussian. It follows that $S_{T}$ is also Gaussian, and therefore we can write

$$
C_{0}=\mathrm{e}^{-r T} \frac{1}{\sqrt{2 \pi \operatorname{Var}\left[S_{T}\right]}} \int_{K}^{\infty}(z-K) \exp \left(-\frac{\left(z-\mathbb{E}\left[S_{T}\right]\right)^{2}}{2 \operatorname{Var}\left[S_{T}\right]}\right) \mathrm{d} z .
$$

Performing this integral, we obtain

$$
C_{0}=\mathrm{e}^{-r T}\left[\sqrt{\frac{\operatorname{Var}\left[S_{T}\right]}{2 \pi}} \exp \left(-\frac{\left(\mathbb{E}\left[S_{T}\right]-K\right)^{2}}{2 \operatorname{Var}\left[S_{T}\right]}\right)+\left(\mathbb{E}\left[S_{T}\right]-K\right) N\left(\frac{\mathbb{E}\left[S_{T}\right]-K}{\sqrt{\operatorname{Var}\left[S_{T}\right]}}\right)\right],
$$

where $N(x)$ is the normal distribution function. Thus, the problem reduces to a determination of the mean and the variance of $S_{T}$. A calculation gives

$$
\mathbb{E}\left[S_{T}\right]=\frac{1}{r}\left[\frac{\kappa}{r+\kappa} \theta+\frac{r}{r+\kappa}\left[\mathrm{e}^{-\kappa T} X_{0}+\theta\left(1-\mathrm{e}^{-\kappa T}\right)\right]\right]
$$

and

$$
\operatorname{Var}\left[S_{T}\right]=\frac{\psi^{2}}{2 \kappa(r+\kappa)^{2}}\left(1-\mathrm{e}^{-2 \kappa T}\right)+z_{T}^{2}\left[\frac{\psi^{2}}{2 r(r+\kappa)^{2}}+\frac{\mathrm{e}^{2 r T}}{\sigma^{2} T}\right] .
$$

Substitution of (53) and (54) in (52) then gives the option price. One can also work out the price processes for options. Similar calculations can be carried out to obtain the prices of futures contracts and futures options. 
Although very simple in its structure, the model we have presented captures nicely certain aspects of the pricing of commodities in an information-theoretic framework. Looking ahead, it would be interesting to pursue a similar line of argument in more complex settings, such as those presented by electricity markets. To get a sense of what is involved in that case see [22]. For an alternative approach to the pricing of commodities in an information-based setting see [23]. For further discussion of the ideas developed in the present paper see [24].

\section{Acknowledgments}

The authors are grateful to participants at the AMaMeF (Advanced Mathematical Methods in Finance) conferences in Alesund, Norway (2009) and Bled, Slovenia (2010), the Workshop on Derivatives Pricing and Risk Management at the Fields Institute, Toronto (2010), and meetings at the University at Warwick (2011) and the London School of Economics (2012), where parts of this work were presented, for helpful comments. We thank the referee for useful suggestions. XY acknowledges support from Shell International Ltd.

[1] I. Karatzas \& S. E. Shreve (1998) Methods of Mathematical Finance. New York: Springer.

[2] D. Duffie (2001) Dynamic Asset Pricing Theory, third edition. Princeton, New Jersey: Princeton University Press.

[3] D. C. Brody, L. P. Hughston \& A. Macrina (2007) Beyond hazard rates: a new framework for credit-risk modelling. In: Advances in Mathematical Finance (M. Fu, R. Jarrow, Ju-Yi Yen \& R. Elliott, eds.), pages 231-257. Basel: Birkhäuser.

[4] D. C. Brody, L. P. Hughston \& A. Macrina (2008) Dam rain and cumulative gain. Proceedings of the Royal Society A 464, 1801-1822.

[5] D. C. Brody, L. P. Hughston \& A. Macrina (2008) Information-based asset pricing. International Journal of Theoretical and Applied Finance 11 (1), 107-142.

[6] A. Macrina (2006) An Information-Based Framework for Asset Pricing: X-Factor Theory and its Applications. PhD thesis, Department of Mathematics, King's College London.

[7] M. Rutkowski \& N. Yu (2007) An extension of the Brody-Hughston-Macrina approach to modelling of defaultable bonds. International Journal of Theoretical and Applied Finance 10 (3), 557-589.

[8] F. Black (1976) The pricing of commodity contracts. J. Fin. Econ. 3, 167-179.

[9] M. Brennan \& E. Schwartz (1985) Evaluating natural resource investments. Journal of Business 58, 135-157.

[10] H. Geman (2005) Commodities and Commodity Derivatives: Modelling and Pricing for Agriculturals, Metals and Energy. Hoboken, New Jersey: John Wiley \& Sons, Inc.

[11] R. Gibson \& E. S. Schwartz (1990) Stochastic convenience yield and the pricing of oil contingent claims. Journal of Finance 45, 959-976.

[12] R. Gibson \& E. S. Schwartz (1991) Valuation of long term oil-linked assets. In: Stochastic Models and Option Values (D. Lund \& B. Øksendal, editors). North Holland: Elsevier.

[13] E. S. Schwartz (1997) The stochastic behavior of commodity prices: implications for valuation and hedging. Journal of Finance 52, 923-973. 
[14] J. E. Hilliard \& J. Reis (1998) Valuation of commodity futures and options under stochastic convenience yields, interest rates, and jump diffusions in the spot. Journal of Financial and Quantitative Analysis 33, 61-86.

[15] J. E. Hilliard \& J. Reis (1999) Jump processes in commodity futures prices and options pricing. American Journal of Agricultural Economics 81, 273-286.

[16] K. Miltersen \& E. S. Schwartz (1998) Pricing of options on commodity futures with stochastic term structure of convenience yields and interest rate. Journal of Financial and Quantitative Analysis 33, 33-59.

[17] E. S. Schwartz \& J. Smith (2000) Short-term variation and long-term dynamics in commodity prices. Management Science 46, 893-911.

[18] K. Miltersen (2003) Commodity price modelling that matches current observables: a new approach. Quantitative Finance 3, 77-105.

[19] M. Nielsen \& E. S. Schwartz (2004) Theory of storage and the pricing of commodity claims. Review of Derivatives Research 7, 5-24.

[20] J. Casassus \& P. Collin-Dufresne (2005) Stochastic convenience yield implied from commodity futures and interest rates. Journal of Finance 58, 2283-2331.

[21] D. Williams (1991) Probability with Martingales. Cambridge University Press.

[22] A. Cartea \& M. G. Figueroa (2005). Pricing in electricity markets: a mean reverting jump diffusion model with seasonality. Applied Mathematical Finance 12 (4), 313-335.

[23] A. Macrina \& J. Sekine (2021) Stochastic modelling with randomized Markov bridges. Stochastics 93 (1), 29-55.

[24] X. Yang (2013) Information-Based Commodity Pricing and the Theory of Signal Processing with Lévy Information. PhD thesis, Department of Mathematics, Imperial College London. 